\title{
Comparison of Intrabeam Scattering Results for a RHIC lattice and a FODO approximation including coupling effects
}

George Parzen

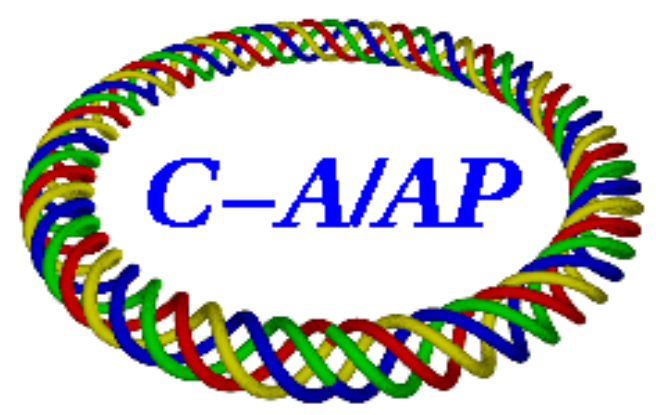

Collider-Accelerator Department

Brookhaven National Laboratory

Upton, NY 11973 


\title{
Comparison of intrabeam scattering results for a RHIC lattice and a FODO approximation including coupling effects
}

\author{
George Parzen
}

October 28, 2004

\begin{abstract}
This note compares the results for the intrabeam scattering growth rates of a Rhic lattice with the growth rates of a similar fodo lattice. The latter lattice has only identical fodo cells and no matching insertions. This comparison will allow us to use the simpler fodo lattice to find more simple analytical results for the intrabeam scattering growth rates. Approximate analytical results for the fodo lattice have been given in [1].
\end{abstract}

\section{Introduction}

This note compares the results for the intrabeam scattering growth rates of a Rhic lattice with the growth rates of a similar fodo lattice. The latter lattice has only identical fodo cells and no matching straight sections. This comparison may allow us to use the simpler fodo lattice to find more simple analytical results for the intrabeam scattering growth rates. Approximate analytical results for the fodo lattice have been given in [1]. The results given below show that the intrabeam scattering growth rates of the fodo lattice can be used to give good approximate results for the intrabeam scattering growth rates of a Rhic lattice for the lattice used in the calculations as presented below..

For the transverse growth rate, $G_{x}$, one can assume that the contribution to $G_{x}$ from the Rhic insertions can be neglected . This assumes that $G_{x}$ 
comes mostly from coupling with $G_{s}$ through the horizontal dispersion which is small in the insertions. One can then find the Rhic growth rate $G_{x, r h i c}$ from the fodo growth rate $G_{x, \text { fodo }}$ using

$$
G_{x, \text { rhic }}=G_{x, \text { fodo }} \frac{L_{\text {fodo }}}{L_{r h i c}}
$$

$L_{\text {fodo }}$, and $L_{r h i c}$ are the lengths of these two lattices respectively.

For the longitudinal growth, the contribution to $G_{s}$ from the Rhic insertions is appreciable but not large. One can write

$$
G_{s, r h i c}=G_{s, f o d o}
$$

with about a $10 \%$ error.

Similar results have been found by A. Fedotov.

\section{The fodo lattice.}

The fodo lattice used below has 72 identical cells, each cell is the same as the cells of the inner arc of a Rhic lattice. This lattice has a length of 2100 meters compared to the Rhic lattice length of 3830 meters.

\section{Computed growth rates for a Rhic lattice and the fodo lattice.}

Growth rates were computed using the IBS.P program [2] for a beam of ionized gold atoms with 1 e9 ions/bunch, using a RF cavuty with $\mathrm{h}=360$ and $\mathrm{V}=300$ volts, and with $\gamma=100$. The growth rates $G_{i}$ are related to the average emittances of the bunch, $\bar{\epsilon}_{i}$ by

$$
G_{i}=\frac{1}{2} \frac{1}{\bar{\epsilon}_{i}} \frac{d \bar{\epsilon}_{i}}{d t} \quad i=x, y, s
$$

Because of the factor of $1 / 2$ in Eq.(3), the growth rates given below are the growth rates for $\sigma$ and not that of the emittance.

The comparison given below for the growth rates for the Rhic and fodo lattices uses the growth rates at $\mathrm{t}=0$, before the emittances have grown. 
For the Rhic lattice the computed results are

$$
\begin{aligned}
& G_{x}=.216 / \text { hour } \\
& G_{s}=4.71 / \text { hour }
\end{aligned}
$$

For the fodo lattice the computed results are

$$
\begin{aligned}
& G_{x}=.351 / \text { hour } \\
& G_{s}=5.29 / \text { hour }
\end{aligned}
$$

$L_{\text {fodo }}=2130 \mathrm{~m}$, and $L_{\text {rhic }}=3834 \mathrm{~m} . \quad L_{\text {fodo }} / L_{r h i c}=.556$. Eq.1 gives $G_{x, r h i c}=.195$ which is in error by $10 \%$. Eq. 2 for $G_{s, r h i c}$ also is off by $10 \%$.

The result for $G_{x}$ depends on how one treats the coupling between the $\mathrm{x}$ and $\mathrm{y}$ motions. A complete treatment of the coupling was given by $\mathrm{A}$. Piwinski [3]. His results are difficult to use as they require knowing all the sources of coupling in the ring such as the skew quadrupoles in each element. What may be a more useable treatment of coupling is the one given in [4]. There it is assumed that the $\mathrm{x}$ and $\mathrm{y}$ motions are completely coupled, which is interperted to mean that

$$
<\epsilon_{x}>=<\epsilon_{y}>
$$

where $<>$ indicates an average over all particles in a bunch. $\epsilon_{x}$ and $\epsilon_{y}$ now are not constants of the motion, and the particle distribution is now assumed to be a function of the total emittance, $\epsilon_{t}=\epsilon_{x}+\epsilon_{y}$ which is a constant of the motion to a fairly good approximation. Equations can then be found for $\left.d<\epsilon_{t}\right\rangle / d t$ and $\left.d<\epsilon_{s}\right\rangle / d t$ which can be integrated to find $\left\langle\epsilon_{t}\right\rangle$ and $<\epsilon_{s}>$ as a function of time. One result of this approach is that the growth rate for $\left\langle\epsilon_{t}\right\rangle, G_{t}$, is related to the growth rates of $\left\langle\epsilon_{x}\right\rangle$ and $\left\langle\epsilon_{y}\right\rangle$ as computed in the absence of coupling, and denoted here by $G_{x}$ and $G_{y}$, by

$$
G_{t}=\frac{1}{2}\left(G_{x}+G_{y}\right)
$$

In the operation of Rhic without electron cooling, the coupling is only about a $10 \%$ effect on the beam size after about an hour [4]. Although the initial growth rate may be reduced by as much as a factor of two, the beam 
size after about an hour is only reduced by about $10 \%$. When electron cooling is present, the possible reduction in the initial growth rate of a factor of 2 , may become more important as the electon cooling has to fight the initial growth rate due to intrabeam scattering.

The above results were found using an older Rhic lattice with 6 insertions each with $\beta=6$ at the crossing points.

\section{Acknowledgements}

I thank Jie Wei and Alexei Fedotov for their helpful comments.

\section{References}

1.J.D. Bjorken and S.K. Mtingwa, Part. Accel.13 (1983) 115, G.Parzen Nuc. Inst. Meth.A256 p.231 (1987), J. Wei PAC (1993) p.3651, J. Wei and G. Parzen PAC (2001), A. Fodotov BNL report C-A/AP No. 168 (2004).

2. G. Parzen Nuc. Inst. Meth. A251 p.220 (1986)

3. A. Piwinski DESY report 90-113 (1990), CERN report 92-01 (1992)

4. G. Parzen Proc, EPAC '88, p.821, (1988) 